\title{
Pregnancy Following in Vitro Fertilization With Minimal Stimulation in a Woman With Diminished Ovarian Reserve: a Case Report and Literature Review
}

\section{Chun Yuan}

Nanjing Medical University

\section{Feiyang Diao}

Nanjing Medical University

Xiang Ma

Nanjing Medical University

Jianyin Liu

Nanjing Medical University

Yan Gao ( $\sim$ gaogao1971@126.com )

Nanjing Medical University

\section{Case report}

Keywords: Follicle-stimulating hormone, in vitro fertilization, minimal stimulation, oral contraceptives, diminished ovarian reserve

Posted Date: May 5th, 2021

DOl: https://doi.org/10.21203/rs.3.rs-476275/v1

License: (c) (i) This work is licensed under a Creative Commons Attribution 4.0 International License. Read Full License 


\section{Abstract}

Background: Among reproductive-aged women with infertility, diminished ovarian reserve (DOR) is an important factor.

Method: we describe a woman with FSH over 20 IU/L who became pregnant through IVF using a minimal stimulation protocol.

Case presentation: Oral contraceptives (OCs) were used before ovarian stimulation, with only one oocyte retrieved. After transfer of one blastocyst to the uterus, she became pregnant and delivered a healthy male infant weighing $3250 \mathrm{~g}$ at 39 weeks' gestational age.

Conclusions: We report this case to declare that minimal stimulation IVF may allow for the possibility of pregnancy in DOR patients, with lower cost and fewer complications.

\section{Background}

Among women of reproductive age, ovarian reserve is a determinant of fertility outcomes. According to previous studies, ovarian reserve can reflect potential reproductive function and oocyte quality.

Diminished ovarian reserve (DOR), in reproductive-aged women, is characterized by regular menses but reduced ovarian response to ovarian stimulation compared to the average. Although the definition of DOR has not been clarified, abnormal basal FSH and AMH values and a decreased AFC are common features of DOR patients in clinical studies.

Although IVF is commonly used for DOR patients, DOR is a major challenge in reproductive medicine and therapeutic regimens [1]. Most reports show that DOR patients have poor IVF results due to a decreased number of oocytes retrieved [2]. Moreover, compared to routine antagonist regimens, the minimal stimulation protocol involves administration of a lower gonadotropin dose and leads to a similar number of retrieved oocytes and rate of pregnancy among DOR patients [3]. Minimal stimulation, which refers to gently controlled ovarian stimulation to obtain 1-6 oocytes, is considered to be a reasonable regimen for DOR patients [4].

In IVF with minimal stimulation, clomiphene citrate (CC) and letrozole have been widely used, due to their reduced cost and lower incidence of ovarian hyperstimulation (OHSS) caused by gonadotropin-induced ovarian hyperstimulation [5]. In contrast to conventional controlled ovarian hyperstimulation $(\mathrm{COH})$, treatment with $\mathrm{CC}$, a selective estrogen receptor modulator, is considered to have a mild stimulatory effect on the ovary. According to a randomized controlled study, the number of retrieved oocytes, total embryos and transferable embryos in IVF patients treated with the standard long regimen was significantly higher than that in IVF patients treated with CC, and the outcomes of the two groups were similar; moreover, the $\mathrm{CC}$ regimen reduced the total dose of gonadotropins and the incidence of OHSS [6]. 
In most women, high FSH levels, low AMH levels and low AFC, which results from an almost exhausted ovarian follicle pool, indicate a significant decrease in fertility [7]. These endocrine variations among reproductive-aged women are primarily related to an obvious decline in the ovarian response and fertility rate [8].

In this report, we present a clinical case of a live birth to an infertile patient with a high FSH level and low $\mathrm{AFC} / \mathrm{AMH}$ values through minimal stimulation IVF and fresh embryo transfer (ET).

\section{Patient And Methods}

\section{Patient history}

This is a case report of a young female patient with endometriosis and decreased ovarian response after surgery in our of reproductive medicine clinical center. The patient was a 29-year-old G0P0 who had a 5year infertility history. Her menses time was regular, at 6-7/27 days, the quantity of bleeding was normal, and she had no dysmenorrhea or dyspareunia. Her husband's serum test showed no irregular findings or irregularities in reproduction function. In 2011, the patient underwent laparoscopic surgery for right ovarian cyst decollement and pelvic adhesiolysis, and a pathological report showed an ovarian endometrioma. On cycle day 3, the AMH level of this patient was $0.61 \mathrm{ng} / \mathrm{ml}$, and there was one antral follicle in the right ovary and one antral follicle in the left ovary observed by ultrasound. The hysterosalpingography report showed that the shape of the uterus was normal, that the right oviduct was partially obstructed, and that the left oviduct was unobstructed. A total of 6 cycles of controlled ovarian stimulation were used, but she failed to become pregnant. She had no other prior surgical history except for breast fibroadenoma surgery (2009) and appendicectomy (2014), with no allergies observed. Any history of sexually transmitted infections and diseases was denied.

\section{Case Presentation}

Failure of ovarian stimulation (first IVF cycle)

In her first cycle of IVF treatment, minimal ovarian stimulation (CC, human menopausal gonadotrophin $(\mathrm{HMG})$ and growth hormone $(\mathrm{GH})$ ) was used. CC $50 \mathrm{mg}$ was taken orally daily for 3 days, and HMG $75 \mathrm{U}$ q.o.d. and GH were taken beginning on day 1 of CC (cycle day 3). On stimulation day 8, two leading follicles developed (mean measurements of $18 \mathrm{~mm}, 14.5 \mathrm{~mm}$ ); her serum estradiol (E2) level was 2271 pmol/L, luteinizing hormone $(\mathrm{LH})$ level was $7.5 \mathrm{IU} / \mathrm{L}$, and progesterone $(\mathrm{P})$ level was $2.05 \mathrm{nmol} / \mathrm{L}$; and her endometrial thickness was $5.6 \mathrm{~mm}$. GnRH-a $0.1 \mathrm{mg}$ was prescribed followed by oocyte retrieval 36 hours later. One oocyte was retrieved, and one embryo was frozen on day 3 due to endometrial factors. In the next cycle (natural cycle, endometrial thickness of $5.6 \mathrm{~mm}$ ), the embryo (at the 14-cell stage) was transferred after assisted hatching. After 14 days, her serum HCG was lower than 5 IU/L.

OC treatment and ovarian stimulation for IVF (Fig. 1) 
Three months later, she began her second cycle of IVF treatment. Her basal FSH was 23.13 IU/L, and after combined oral contraceptives (OCs) for 7, 7, 12, 7, and 7 days (total 40 days) were used, her FSH levels changed to $32.22,27.26,28.25,19.50$, and $15.82 \mathrm{IU} / \mathrm{L}$, respectively. On day 40 of OCs, she was treated with CC 50 mg, HMG $75 \mathrm{IU}$ and GH $4 \mathrm{U}$ daily. On stimulation day 10, the leading follicles developed with mean diameters of $18.5,13.5$ and $12.5 \mathrm{~mm}$, and her serum LH level was $16.31 \mathrm{IU} / \mathrm{L}$, E2 level was 5358.38 $\mathrm{pmol} / \mathrm{L}, \mathrm{P}$ level was $1.80 \mathrm{nmol} / \mathrm{L}$, and endometrial thickness was $4.8 \mathrm{~mm}$. GnRH-a $0.1 \mathrm{mg}$ was prescribed followed by oocyte retrieval 36 hours later. Only one oocyte was retrieved. According to the doctor's opinion, the embryo was grown into a 3CC-grade blastocyst (Fig. 2). Five days after the day of ovum pick up (DOPU), her endometrial thickness measured $4.2 \mathrm{~mm}$, and ET was performed without any difficulty, with the $17.3 \mathrm{~cm}$ COOK Embryo Transfer Catheter to the fundus of uterus. Luteal support was applied with oral E2 2 mg daily, oral dydrogesterone $20 \mathrm{mg}$ daily and micronized P $100 \mathrm{mg}$ vaginally twice a day during pregnancy until 90 days of gestation. Her serum beta-HCG at 14 days after ET was 2893 IU/L. A single intrauterine gestational sac was observed at a gestational age of 47 days ( $6^{+5}$ weeks). At 39 weeks' gestation, she received a cesarean section, and a healthy male infant weighing $3250 \mathrm{~g}$ was delivered.

\section{Discussion And Conclusion}

Over the last decade, there have been few reports on successful live births from minimal stimulation IVF in a DOR patient using OCs to decrease high FSH levels. In addition to high FSH levels, the patient had extremely low AMH/AFC values and a long infertility history. According to the opinions of most clinical investigators, advanced maternal age in IVF is not recommended and is usually accompanied by several social issues and possible gestational complications [9]. However, compared to women of advanced age, young patients with DOR always have strong willingness for child-bearing. Based on the advantage of lower cost and fewer complications, IVF with a mild/minimal stimulation protocol is considered appropriate for infertile patients, especially DOR patients [10].

DOR patients are a group of infertile women who are characterized by poor ovarian response (POR), which leads to poor ART outcomes, such as few retrieved oocytes, few obtained embryos, and low pregnancy rates [11]. In 2011, the European Society of Human Reproduction and Embryology (ESHRE) published a consensus named the Bologna criteria to describe the clinical manifestation of POR. This consensus defined the criteria for POR as follows: advanced age or other risk factors; a history of $\leq 3$ oocytes with conventional stimulation; and an abnormal ovarian reserve test [12]. Although the definition of DOR remains blurry, the ESHRE Bologna criteria for POR could make the diagnosis of DOR clear [1, 13]. Over the last few decades, there have been many clinical investigations on IVF that have analyzed the current situation in DOR patients. A minimal stimulation protocol in IVF has been used to improve the efficiency of gonadotropin and pregnancy outcomes in DOR patients. Pilehvari et al. reported that there was no significant difference in pregnancy rates between the minimal stimulation IVF group and the conventional stimulation IVF group in a randomized controlled trial [3]. Through a retrospective cohort 
study, Lazer et al. reported their result showing that the minimal stimulation protocol in IVF resulted in a significantly higher pregnancy rate and live birth rate than high-dose stimulation in women with POR [11].

CC and low-dose gonadotropin are commonly applied in IVF with minimal stimulation protocols [14]. As a kind of selective estrogen receptor modulator, CC can inhibit the estrogen feedback system by binding to estrogen receptors in the hypothalamus and then stimulate the pituitary to secrete more FSH [15]. In Beverly GR et al.'s report, endometrial thickness was influenced during IVF by the CC-minimal stimulation protocol, but no negative results were observed in the subsequent frozen embryo transfer (FET) cycle [16]. In this case, the patient received fresh ET due to financial factors.

In this case, we show that OCs are effective in decreasing high FSH levels. In recent decades, only a few studies focusing on the OC effect of IVF with minimal stimulation protocols have been reported. Emmett $\mathrm{F}$ et al. presented evidence showing that OC pretreatment before IVF with a minimal stimulation protocol played an important role in LH suppression, and the pregnancy rates were similar to those of conventional stimulation IVF [10]. Similar to CC, OCs exhibit obvious features of low cost and accessibility for patients.

In conclusion, clinicians should consider minimal stimulation IVF to be a frequently used regimen for DOR patients. This case provides evidence that DOR patients with extremely high FSH and low AMH levels achieve a positive result from IVF; however, more clinical experience with minimal stimulation IVF needs to be accumulated.

\section{Abbreviations}

IVF in vitro fertilization

FSH follicle-stimulating hormone

DOR diminished ovarian reserve

AMH anti-Mullerian hormone

AFC antral follicle count

OCs oral contraceptives

CC clomiphene citrate

ET embryo transfer

HMG human menopausal gonadotrophin

LH luteinizing hormone 


\section{Declarations}

Acknowledgments:

We obtained informed consent from the patient, and no identity information is provided in this report, to protect the privacy of the patient.

\section{Founding:}

This study was supported by research grants from the National Basic Research Program of China, National Natural Science Foundation of China (81901449), Province Basic Research Program of Jiangsu, Province Natural Science Foundation of Jiangsu (BK20191076) and State Key Laboratory Foundation of Reproductive Medicine (SKLRM-K201805).

\section{Competing interest:}

The authors declare that they have no competing interests.

\section{Authors' contribution:}

Study concept and design: Chun Yuan and Yan Gao. Acquisition of data: Yan Gao, Jiayin Liu. Writing of the article: Chun Yuan. Critical revision of the article: Feiyang Diao, Xiang Ma. All authors agree with the article's results and conclusions. All authors have read, and confirm that they meet, the authorship criteria.

\section{Ethics approval and consent to participate:}

This study was approved by the Ethical Committee of Hospital.

\section{Availability of data and material:}

Data sharing not applicable to this study as no datasets were generated or analysed during the current study.

\section{Disclosure Summary:}

The authors have nothing to disclose.

\section{References}

1. Cohen J, Chabbert-Buffet N, Darai E. Diminished ovarian reserve, premature ovarian failure, poor ovarian responder--a plea for universal definitions. Journal of assisted reproduction and genetics. 2015;32(12):1709-1712. https://doi.org/ 10.1007/s10815-015-0595-y.

2. Practice Committee of the American Society for Reproductive M. Testing and interpreting measures of ovarian reserve: a committee opinion. Fertility and sterility. 2012;98(6):1407-1415. https://doi.org/10.1016/j.fertnstert.2012.09.036. 
3. Pilehvari S, ShahrokhTehraninejad E, Hosseinrashidi B, Keikhah F, Haghollahi F, Aziminekoo E. Comparison Pregnancy Outcomes Between Minimal Stimulation Protocol and Conventional GnRH Antagonist Protocols in Poor Ovarian Responders. Journal of family \& reproductive health. 2016;10(1):35-42.

4. Zarek SM, Muasher SJ. Mild/minimal stimulation for in vitro fertilization: an old idea that needs to be revisited. Fertility and sterility. 2011;95(8):2449-2455. https://doi.org/10.1016/j.fertnstert.2011.04.041.

5. Haas J, Casper RF. In vitro fertilization treatments with the use of clomiphene citrate or letrozole. Fertility and sterility. 2017;108(4):568-571. 10.1016/j.fertnstert.2017.08.017

6. Karimzadeh MA, Ahmadi S, Oskouian H, Rahmani E. Comparison of mild stimulation and conventional stimulation in ART outcome. Archives of gynecology and obstetrics. 2010;281(4):741746. https://doi.org/10.1007/s00404-009-1252-4.

7. te Velde ER, Pearson PL. The variability of female reproductive ageing. Human reproduction update. 2002;8(2):141-154. https://doi.org/10.1093/humupd/8.2.141.

8. Khan HL, Bhatti S, Suhail S, Gul R, Awais A, Hamayun H, Enver F, Abbas S, Hassan Z, Nisar R, Sardar $S$, Asif W. Antral follicle count (AFC) and serum anti-Mullerian hormone (AMH) are the predictors of natural fecundability have similar trends irrespective of fertility status and menstrual characteristics among fertile and infertile women below the age of 40 years. Reproductive biology and endocrinology : RB\&E. 2019;17(1):20. https://doi.org/10.1186/s12958-019-0464-0.

9. Ubaldi FM, Cimadomo D, Vaiarelli A, Fabozzi G, Venturella R, Maggiulli R, Mazzilli R, Ferrero S, Palagiano A, Rienzi L. Advanced Maternal Age in IVF: Still a Challenge? The Present and the Future of Its Treatment. Frontiers in endocrinology. 2019;10:94. https://doi.org/10.3389/fendo.2019.00094.

10. Branigan EF, Estes MA. Minimal stimulation IVF using clomiphene citrate and oral contraceptive pill pretreatment for LH suppression. Fertility and sterility. 2000;73(3):587-590. https://doi.org/10.1016/s0015-0282(99)00584-1.

11. Lazer T, Dar S, Shlush E, Al Kudmani BS, Quach K, Sojecki A, Glass K, Sharma P, Baratz A, Librach CL. Comparison of IVF Outcomes between Minimal Stimulation and High-Dose Stimulation for Patients with Poor Ovarian Reserve. International journal of reproductive medicine. 2014;2014:581451. https://doi.org/10.1155/2014/581451.

12. Ferraretti AP, La Marca A, Fauser BC, Tarlatzis B, Nargund G, Gianaroli L, Definition EwgoPOR. ESHRE consensus on the definition of 'poor response' to ovarian stimulation for in vitro fertilization: the Bologna criteria. Human reproduction. 2011;26(7):1616-1624. https://doi.org/10.1093/humrep/der092.

13. Pastore LM, Christianson MS, Stelling J, Kearns WG, Segars JH. Reproductive ovarian testing and the alphabet soup of diagnoses: DOR, POI, POF, POR, and FOR. Journal of assisted reproduction and genetics. 2018;35(1):17-23.https://doi.org/10.1007/ s10815-017-1058-4.

14. Reed BG, Babayev SN, Bukulmez O. Shifting paradigms in diminished ovarian reserve and advanced reproductive age in assisted reproduction: customization instead of conformity. Seminars in 
reproductive medicine. 2015;33(3):169-178. https://doi.org/10.1055/s-0035-1552585.

15. Haritha S, Rajagopalan G. Follicular growth, endometrial thickness, and serum estradiol levels in spontaneous and clomiphene citrate-induced cycles. International journal of gynaecology and obstetrics: the official organ of the International Federation of Gynaecology and Obstetrics. 2003;81(3):287-292. https://doi.org/10.1016/s0020-7292(02)00404-6.

16. Reed BG, Wu JL, Nemer LB, Carr BR, Bukulmez O. Use of Clomiphene Citrate in minimal stimulation in vitro fertilization negatively impacts endometrial thickness: an argument for a freeze-all approach. JBRA assisted reproduction. 2018;22(4):355-362. https://doi.org/10.5935/1518-0557.20180070.

\section{Figures}

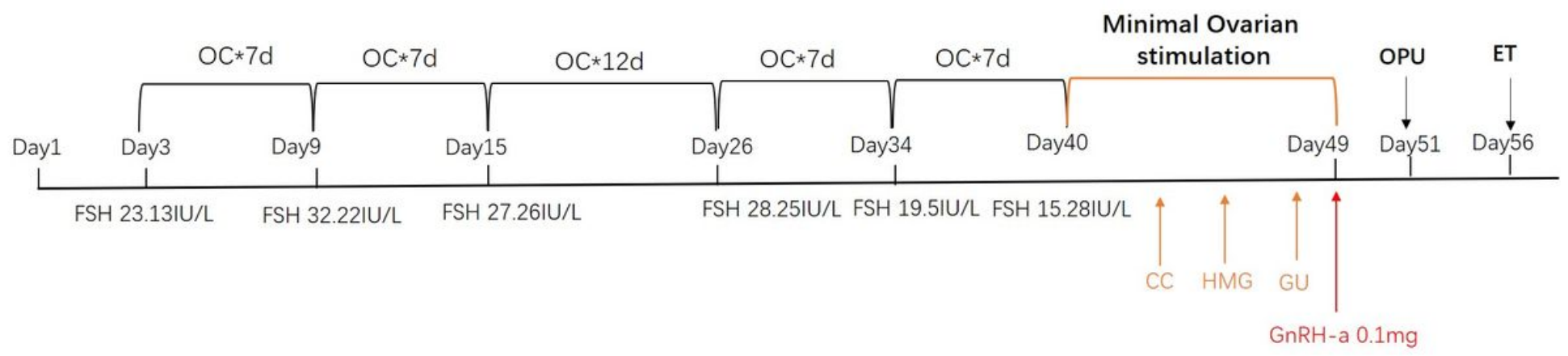

\section{Figure 1}

The procedure of OC-IVF in this case 


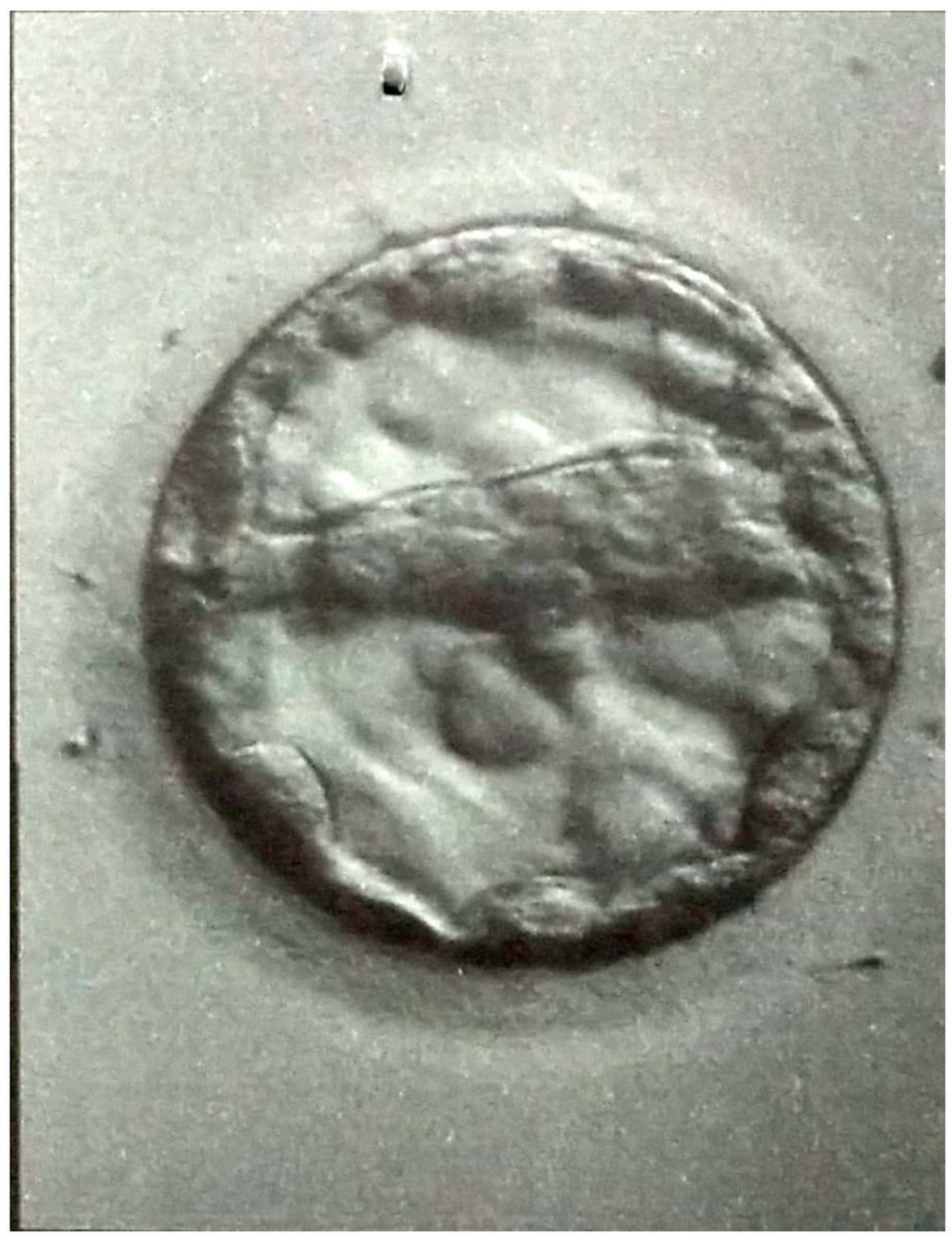

Figure 2

3CC grade blastocyst transferred on day 5 . 